\title{
Theranostics
}

Research Paper

$2011 ; 1: 240-250$

\section{Folic Acid-conjugated Graphene Oxide loaded with Photosensitizers for Targeting Photodynamic Therapy}

\section{Peng Huang, Cheng Xu, Jing Lin, Can Wang, Xiansong Wang, Chunlei Zhang, Xuejiao Zhou, Shouwu Guo, Daxiang Cui ${ }^{凶}$}

National Key Laboratory of Nano/Micro Fabrication Technology, Key Laboratory for Thin Film and Microfabrication of Ministry of Education, Institute of Micro-Nano Science and Technology, Shanghai Jiao Tong University, 800 Dongchuan Road, Shanghai 200240, PR China

$\triangle$ Corresponding author: Tel/Fax: +86 21 34206886. E-mail: dxcui@sjtu.edu.cn (Daxiang Cui)

(C) Ivyspring International Publisher. This is an open-access article distributed under the terms of the Creative Commons License (http://creativecommons.org/ licenses/by-nc-nd/3.0/). Reproduction is permitted for personal, noncommercial use, provided that the article is in whole, unmodified, and properly cited.

Received: 2011.03.18; Accepted: 2011.04.11; Published: 2011.04.13

\begin{abstract}
Photodynamic therapy (PDT) has emerged as an alternative and promising noninvasive treatment for cancer as well as non-cancer diseases, which involves the uptake of photosensitizers (PSs) by cancer cells followed by irradiation. The use of nanomaterials as carriers of PSs is a very promising approach to improve the development of PDT in clinical medicine. In this study, a novel folic acid-conjugated graphene oxide (GO) was strategically designed and prepared as targeting drug delivery system to achieve higher specificity. The second generation photosensitizer (PS) Chlorin e6 (Ce6) was effectively loaded into the system via hydrophobic interactions and $\Pi-\pi$ stacking. The nanocarriers can significantly increase the accumulation of $\mathrm{Ce} 6$ in tumor cells and lead to a remarkable photodynamic efficacy on MGC803 cells upon irradiation. These suggested that folic acid-conjugated GO loaded Ce6 had great potential as effective drug delivery system in targeting PDT.
\end{abstract}

Key words: Photodynamic therapy, Graphene oxide, Folic acid, Chlorin e6, Drug delivery.

\section{Introduction}

Photodynamic therapy (PDT) is an increasingly recognized alternative to treat various cancers in clinical practice [1]. Compared with conventionally therapeutic modalities (chemotherapy, surgery, or radiotherapy), PDT has the following advantages [2-3]: (1) the minimal invasive nature, (2) the selectivity of the interest area, (3) the ability to treat patients with repeated doses without initiating resistance or exceeding total dose limitations (as associated with radiotherapy). PDT involves the systemic, local, or topical administration of photosensitizers (PSs), and then irradiates using light of appropriate wavelength and dosage, and finally generates reactive oxygen species (ROSs) that induce cell death and necrosis of tumor components [4-5].
The most commonly used PSs are porphyrin-based molecules [6]. Many of them are limited because of prolonged cutaneous photosensitivity, poor water solubility and inadequate selectivity [7], which are encountered in clinical applications of numerous traditional chemicals [8-10]. Therefore, numerous approaches have been proposed to incorporate PSs into various delivery carriers such as liposome, polymer nanoparticles, gold nanoparticles, quantum dots, magnetic nanoparticles, silica nanoparticles, and so on [11-14], which realize high selectivity, high efficacy and low risk of adverse events.

Carbon-based nanomaterials, including fullerenes [15], carbon nanotubes [16-17], carbon nanohorns [18], and their derivatives, exhibit great application 
prospects in PDT. For example, Fullerenes and their derivatives, due to their extended $\Pi$-conjugation, have been reported as PSs in PDT [19-20]. Tan group have engineered a novel molecular complex of a PS, an ssDNA aptamer, and single-walled carbon nanotubes (SWNTs) for controllable singlet oxygen $\left({ }^{1} \mathrm{O}_{2}\right)$ generation [21]. We also have explored polyamidoamine dendrimer modified multi-walled carbon nanotubes as new vehicles to deliver 5-aminolevulinic acid (5-ALA) to tumor MGC803 cells [22]. Zhang et al. fabricated Zinc phthalocyanine $(\mathrm{ZnPc})$ and bovine serum albumin (BSA) co-loaded by single-wall carbon nanohorns with holes opened (SWNHox) to achieve a double PDT and photohyperthermia (PHT) cancer phototherapy system that uses a single laser [23]. Therefore, carbon-based nanomaterials have highlighted the direction as efficient delivery systems in PDT.

Graphene, a very recent rising star, with an atomically thin, 2D honeycomb lattice that consists of $\mathrm{sp}^{2}$-hybridized carbons, exhibits remarkable electronic, thermal, optical, and mechanical properties [24-25]. Particularly, graphene oxide (GO), graphene's water-soluble derivative, has been found important potential applications in drug delivery [26-28] and enzyme immobilization [29] due to their large specific surface area and abundant functional groups (epoxide, hydroxyl, and carboxylic groups). Dai group for the first time employed PEG modified nanoscale GO $(<50 \mathrm{~nm})$ as drug carriers to load doxorubicin (DOX) via noncovalent physisorption (ח-stacking)[26] and camptothecin(CPT) analogue SN38 via noncovalent van der Waals interaction [27]. Whereafter, Chen group prepared GO-doxorubicin hydrochloride nanohybrid and further investigated the loading and release behaviors of doxorubicin hydrochloride in the nanohybrid system [30]. Zhijun Zhang et al fabricated folic acid-conjugated nanoscale GO to control co-loading of DOX and CPT, and demonstrated that the system shows specific targeting to MCF-7 cells and remarkably high cytotoxicity [28]. Recently, Zhuang Liu et al. found that nanographene sheets seem to home in on tumors, and can heat up and kill them from within with the help of a laser [31]. However, the controlled loading and targeted delivery PSs by GO-based drug carriers remain unexplored.

Herein, we designed and prepared folic acid-conjugated GO as drug delivery system for targeting PDT. In our strategy, sulfonic acid groups were introduced to GO, which rendered it stable under physiological conditions, and folic acid (FA) molecules were conjugated with the GO for targeting specific cells with folate receptors. Furthermore, controlled loading and targeted delivery PSs using the
GO as a carrier were investigated. Chlorin e6 (Ce6), a promising PS with a high sensitizing efficacy, was loaded onto the GO in a controlled way. To our knowledge, this is the first report on the controlled loading and targeted delivery of $\mathrm{Ce} 6$ on graphene-based carriers (Scheme 1/Fig. A).

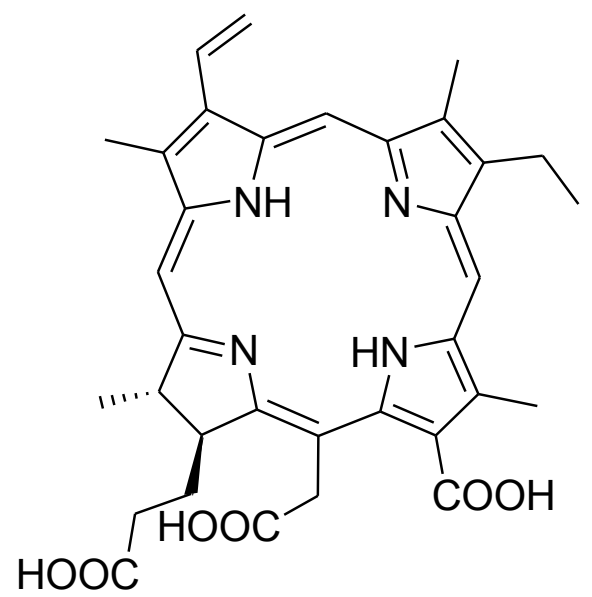

Fig. A. Scheme I Chemical structure of Ce6.

\section{Methods}

\section{Synthesis of graphene oxide}

GO was prepared using nature graphite powders as the raw materials by a modified Hummers method [32-34]. Briefly, $2 \mathrm{~g}$ of graphite and $1 \mathrm{~g}$ of $\mathrm{NaNO}_{3}$ were put into a flask at $0{ }^{\circ} \mathrm{C}$. Then, $50 \mathrm{~mL}$ of concentrated $\mathrm{H}_{2} \mathrm{SO}_{4}$ was added to the mixture, which was stirred for $30 \mathrm{~min}$ at $5{ }^{\circ} \mathrm{C}$. Subsequently, $7 \mathrm{~g}$ of $\mathrm{KMnO}_{4}$ was added to the reaction system step-wisely over $1 \mathrm{~h}$, meanwhile the temperature of the mixture was kept below $20^{\circ} \mathrm{C}$. Then, the temperature was lifted to $35^{\circ} \mathrm{C}$ and stirred for $2 \mathrm{~h}$. Afterwards, $90 \mathrm{~mL}$ of deionized distilled (DD) water was slowly added into the solution, and the temperature of the reaction system jumped to $70{ }^{\circ} \mathrm{C}$ instantly. Finally, $7 \mathrm{~mL}$ of $\mathrm{H}_{2} \mathrm{O}_{2}$ $(30 \%)$ and $55 \mathrm{~mL}$ of DD water were poured into the reaction system, resulting in the formation of bright yellow suspension. The GO was separated by filtration, washed for three times with diluted $\mathrm{HCl}(3 \%)$, and then dispersed in DD water. Exfoliation of GO was approached by sonicating $(200 \mathrm{w})$ the GO in DD water at room temperature for $1 \mathrm{~h}$ generating homogeneous GO dispersions.

\section{Conjugation of folic acid with graphene oxide (FA-GO)}

FA molecules were conjugated to the GO according to the literature [28]. Briefly, $5 \mathrm{~g}$ of $\mathrm{NaOH}$ and $5 \mathrm{~g}$ of $\mathrm{ClCH}_{2} \mathrm{COONa}$ were added to $100 \mathrm{~mL}$ of $\mathrm{GO}$ 
solution $(1 \mathrm{mg} / \mathrm{mL})$. After sonicated for $2 \mathrm{~h}$, the resulting product $(\mathrm{GO}-\mathrm{COOH})$ was neutralized with dilute $\mathrm{HCl}$ and washed for five times with DD water by centrifugation. Then, the GO-COOH suspension was dialyzed against DD water for over $48 \mathrm{~h}$ to remove any ions. Afterwards, $200 \mathrm{mg}$ sulfanilic acid and $80 \mathrm{mg}$ sodium nitrite were dissolved in $20 \mathrm{~mL}$ of $0.25 \% \mathrm{NaOH}$. The mixture solution was added dropwise to $26 \mathrm{~mL}$ of $0.1 \mathrm{~N} \mathrm{HCl}$ solutions in an ice bath. The diazonium salt solution was added to the dispersion of GO-COOH in an ice bath with stirring for $2 \mathrm{~h}$. After dialyzing against DD water for over $48 \mathrm{~h}$, the sulfonated $\mathrm{GO}-\mathrm{COOH}\left(\mathrm{GOSO}_{3} \mathrm{H}\right)$ was stored at $4{ }^{\circ} \mathrm{C}$. The $\mathrm{GO}-\mathrm{SO}_{3} \mathrm{H}$ was then conjugated with $\mathrm{FA}$ using a modification of the standard EDC-NHS reaction as described by Jönsson et al [17, 35]. $100 \mathrm{mg}$ of GO-SO ${ }_{3} \mathrm{H}$ dispersion was activated by an EDC/NHS solution and treated by ultrasonication for $2 \mathrm{~h}$. Finally, $20 \mathrm{~mL}$ of $0.5 \%$ FA was added to form a mixed solution and allowed to react at room temperature for $12 \mathrm{~h}$. The unreacted materials were separated out by dialysis against sodium bicarbonate solution ( $\mathrm{pH}$ 8.0) for 48 $\mathrm{h}$, followed by dialysis against DD water for $24 \mathrm{~h}$.

\section{Photosensitizers Ce6 loaded by FA-GO (FA-GO-Ce6) (Scheme 2/Fig. B)}

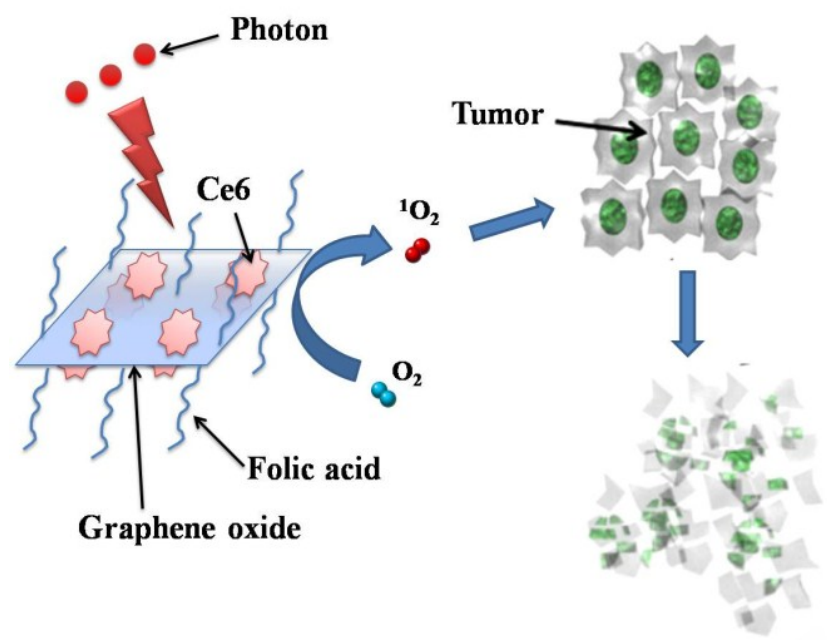

Fig. B. Scheme 2. Photosensitizers Ce6 loaded by folic acid-conjugated graphene oxide.

Ce6 was dissolved in DD water and mixed with FA-GO aqueous suspension $(0.5 \mathrm{mg} / \mathrm{mL})$ at room temperature for $24 \mathrm{~h}$. Then the whole system was dialyzed against DD water for $24 \mathrm{~h}$. The standard curve was established in one range of drug concentration. UV-vis measurements of FA-GO-Ce6 in the mixed solvent $(0.1 \mathrm{~mL}$ ethanol $+2.9 \mathrm{~mL} \mathrm{DD})$ were carried out. The loading efficiency of Ce6 was calculated ac- cording to UV absorbance at $663 \mathrm{~nm}$. Every experiment was repeated for three times.

\section{Characterization}

Atomic force microscopic (AFM) images were taken on a MultiMode Nanoscope V scanning probe microscopy (SPM) system (Veeco, USA). Transmission electron microscopy (TEM) images were acquired using a JEM-2010 (JEOL Ltd., Japan) operated at an accelerating voltage of $200 \mathrm{kV}$. Scanning electron microscopy (SEM) images were obtained with FESEM: ZEISS operated at $5.0 \mathrm{kV}$. UV-vis spectra were measured at $20^{\circ} \mathrm{C}$ with a Shimadzu UV-2450 UV-visible spectrophotometer equipped with a 10-mm quartz cell, where the light path length was 1 $\mathrm{cm}$. Fluorescence spectra were recorded on a HITACHIH FL-4600 spectrofluorimeter. The fourier transform infrared (FTIR) spectra were recorded on a Bruker EQUINOX 55 FTIR Spectrometer. The surface charge of samples was measured with Zeta potential measurements in water (NICOMP 380ZLS Zeta potential/Particle sizer).

\section{Cellular uptake in vitro}

Human stomach cancer MGC803 cells $\left(3 \times 10^{3}\right.$ cells per well) were seeded in 96-well plates and divided into three groups: (1) Cells were treated with GO-Ce6 in RPMI-1640 medium without FA. (2) Cells were treated with FA-GO-Ce6 in RPMI-1640 medium with FA. (3) Cells were treated with FA-GO-Ce6 in RPMI-1640 medium without FA. All Cells were incubated overnight at $37{ }^{\circ} \mathrm{C}$ in a humidified $5 \% \mathrm{CO}_{2}$ atmosphere, and continued to culture for 1 day. After being rinsed with PBS ( $\mathrm{pH} 7.4)$, the cells were incubated with $100 \mu \mathrm{L} 50 \mu \mathrm{g} / \mathrm{mL}$ of different composites for $30 \mathrm{~min}$ at $37^{\circ} \mathrm{C}$ in the dark under the same conditions. After the wells were washed three times and monitored by fluorescence microscopy (NIKON ECLIPSE TS100) and CRi Nuance multispectral imaging systems (Cambridge Research \& Instrumentation, Inc., Woburn, MA, USA). A spectral cube for cells, which contains the complete spectral information at 10-nm wavelength intervals from 520 to 720 nm were collected by CRi Nuance systems.

MGC803 cells incubated with FA-GO-Ce6 for 24 $\mathrm{h}$ were washed with PBS and fixed in $4 \%$ glutaraldehyde solution for $1 \mathrm{~h}$ at $4{ }^{\circ} \mathrm{C}$. The cells were postfixed in $1 \%$ osmium tetroxide for $1 \mathrm{~h}$ at room temperature and washed. Then, cells were scraped and concentrated in $2.5 \%$ agar in $0.05 \mathrm{M}$ cacodylate buffer. The obtained samples were then treated with $2 \%$ uranyl acetate solution for $1 \mathrm{~h}$ and subsequently dehydrated by means of ethanol/water solutions, with increasing ethanol content and embedded in epoxy resin. The 
samples were cut at $60 \mathrm{~nm}$ (ultrathin sections) with an ultramicrotome. Ultrathin sections were transferred to the 300 mesh copper grid and stained with 5\% uranyl acetate. The copper grid was observed on a transmission electron microscope (TEM, Joel JEM-1230) at 100 $\mathrm{kV}$.

\section{Cytotoxicity assessment}

MGC803 cells $\left(3 \times 10^{3}\right.$ cells per well) were seeded in 96-well plates and incubated overnight at $37^{\circ} \mathrm{C}$ in a humidified $5 \% \mathrm{CO}_{2}$ atmosphere. After being rinsed with PBS ( $\mathrm{pH}$ 7.4), the cells were incubated with 100 $\mu \mathrm{L}$ of varying concentration of different nano-composites prepared above for $24 \mathrm{~h}$ at $37^{\circ} \mathrm{C}$ in the dark under the same conditions. Then cells were rinsed with PBS and incubated another $48 \mathrm{~h}$. Cell viability was determined by the colorimetric 3-(4, 5-dimethylthiazol-2-yl)-2, 5-diphenyltetrazolium bromide (MTT) assay [36-38]. Cells were rinsed with PBS and then incubated with culture medium containing $0.5 \mathrm{mg} \mathrm{mL}^{-1} \mathrm{MTT}$ reagent for $3 \mathrm{~h}$. The medium was then removed and the formazan crystals formed were dissolved in $100 \mu \mathrm{L}$ of DMSO. The absorbance at $492 \mathrm{~nm}$ for each well was recorded by a microplate reader.

\section{In vitro PDT effects}

Two 96-well plates were set up as dark control and experimental group and these plates were seeded, exposed identically to the plates prepared for the cytotoxicity assessment. The cells in experimental group were then rinsed again with PBS and immersed in $100 \mu \mathrm{L}$ of fresh culture medium before being irradiated using a $632.8 \mathrm{~nm} \mathrm{He}-\mathrm{Ne}$ laser with energy density of $\sim 30 \mathrm{~mW} / \mathrm{cm}^{2}$ for $10 \mathrm{~min}$. After irradiation, cells were incubated $48 \mathrm{~h}$ in a $5 \% \mathrm{CO}_{2}, 95 \%$ air humidified incubator at $37^{\circ} \mathrm{C}$. Dark control group keeps identical to experimental group without irradiation. PDT effect assay was also determined by MTT assay.

\section{Statistical analysis}

All data are presented in this article as mean result \pm SD. Statistical differences were evaluated using the $t$ test and considered significant at $P<0.05$ level. All figures shown in this article were obtained from three independent experiments with similar results.

\section{Results}

\section{Synthesis and characterization of GO and FA-GO}

GO was prepared using nature graphite powders as the raw materials through a modified Hummers method [32-34]. The morphology of as-prepared GO was characterized by AFM (Figure 1a), TEM (Figure 1c), and SEM (Figure 1d).



Fig. I (a) A tapping mode AFM image of graphene oxide (GO) sheets on mica surface, (b) the height profile of the AFM image, (c) TEM image of the GO, and (d) SEM image of the GO. 
The results indicated that the GO existed in the sheet-like shapes. The thickness, measured from the height profile of the AFM image, Fig. $1 b$, is about 1.2 $\mathrm{nm}$, which is consistent with the data reported in the literature [32], indicating that the formation of single layered GO.

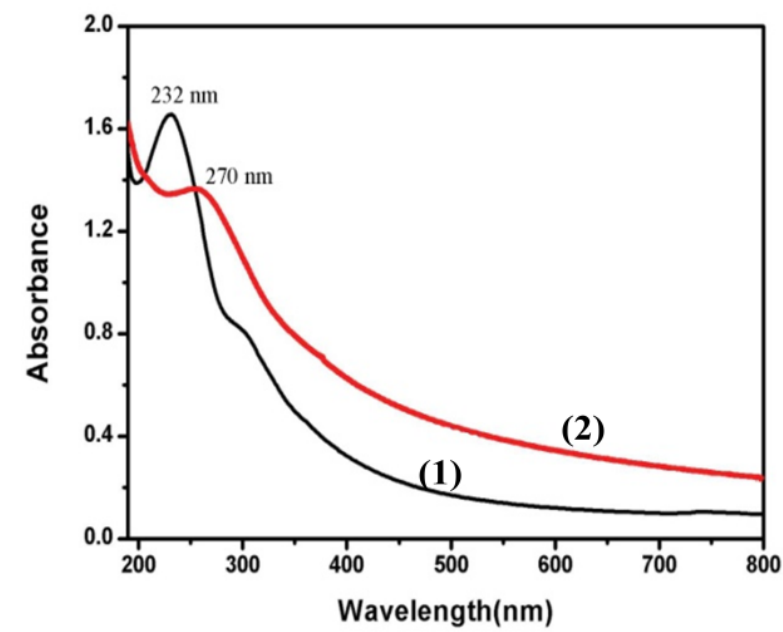

Fig. 2 UV-vis spectra of the GO (I) and FA-GO (2) in aqueous solution.

The as-prepared GO was also determined by FT-IR and UV-vis spectroscopy. The FT-IR spectrum of GO showed that there are ester, hydroxyl, and epoxide groups in the GO sheets (Supplementary Material: Fig. S1) [29]. The raman spectrum of GO showed that there are two special peaks: $G$ (the $E_{2 g}$ mode of $\mathrm{sp}^{2}$ carbon atoms) and $\mathrm{D}$ (the symmetry $A_{1 g}$ mode), indicating that the prepared GO were of high purity (Supplementary Material: Fig. S2) FA mole- cules were conjugated to the GO according to the literature [28]. One can see that a peak at $232 \mathrm{~nm}$ disappears while a new peak at $270 \mathrm{~nm}$ appears due to the presence of FA in the FA-GO (Fig. 2).

\section{Spectroscopic properties of FA-GO-Ce6}

We investigated the binding of Ce6 to FA-GO. The second generation PS Ce6, was selected because of its high photosensitizing efficacy and low dark toxicity, and has been widely incorporated into drug carriers for PDT [39-40]. Optical absorption and fluorescence spectroscopy were used to analyze the interactions between FA-GO and Ce6. Fig. 3(a) shows the absorption spectra of FA-GO, Ce6, and FA-GO-Ce6 in water. FA-GO without $\mathrm{Ce} 6$ show virtually no absorption in the range of $600 \sim 800 \mathrm{~nm}$. The Ce6 spectrum exhibits a strong Soret absorption at 406 $\mathrm{nm}$, and weak Q-bands between 500 and $700 \mathrm{~nm}$. UV-vis spectrum of FA-GO-Ce6 revealed Ce6 peaks superimposing with the absorption curve of FA-GO, suggesting loading of Ce6 onto FA-GO, and also indicating that there is no changes in the Ce6 chromophore after carried. Fig. 3(b) represents the fluorescence emission spectra of aqueous solution of FA-GO, Ce6, and FA-GO-Ce6. The fluorescence emission spectra of Ce6 and FA-GO-Ce6 at the same Ce6 concentration are similar with maximum emission at $\sim 652 \mathrm{~nm}$. The results also show drastic fluorescence quenching of Ce6 in the FA-GO-Ce6 case, suggesting close proximity of Ce6 to the FA-GO sheets. We suggest that the binding of Ce6 onto FA-GO was noncovalent in nature, driven by hydrophobic interactions and п-п stacking between Ce6 and aromatic regions of the GO sheets.
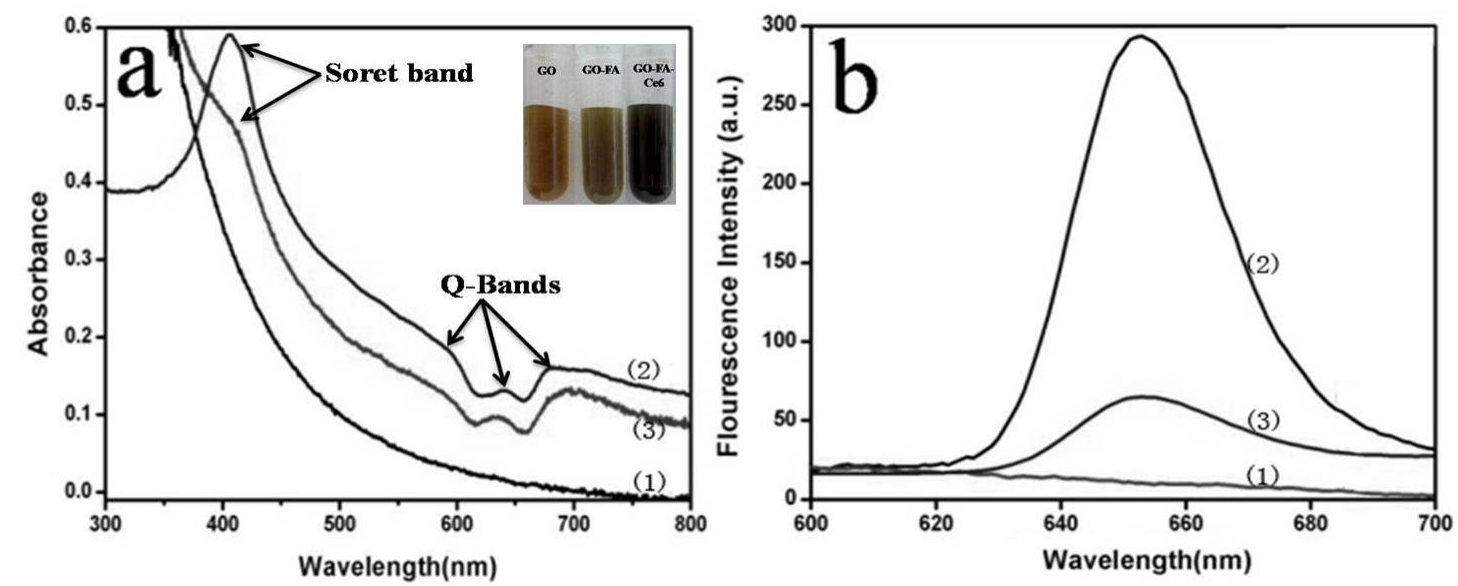

Fig. 3 Absorption spectra (a) and Fluorescence emission spectra (b) of FA-GO (I), Chlorin e6 (Ce6) (2), and FA-GO-Ce6(3) in water (The inset is the photographs of GO, FA-GO, and FA-GO-Ce6 in tubes). 


\section{Zeta potential measurements}

To further study the interaction mechanism between Ce6 and GO, a series of concentrations of GO were chosen to interact with $\mathrm{Ce} 6$ and the resultant products were monitored by Zeta potential measurement and fluorescence spectroscopy. Zeta potentials and illustration of formation mechanism of the GO-Ce6 composite suspensions as a function of the component ratio were shown in Fig. 4. An aqueous suspension of GO exhibits a zeta potential of ca. -40.28 , indicative of negatively charged surfaces caused by the presence of hydrophilic carboxyl groups. With the increase of $\mathrm{Ce} 6$ concentration, the zeta potential of the GO-Ce6 composites becomes more negative (-50.69) due to the aggregation of negatively charged Ce6 on the surface of GO. Each Ce6 molecule has three carboxyl groups [21], which might improve the water solubility of the complex of Ce6 and FA-GO. We suggested that there are two different hybrid structures in the solution, including GO-Ce6-GO and Ce6-GO-Ce6 sandwiches, depending on the concentration of Ce6 (see Fig. 4). Excitation of the GO-Ce6 composites and the control sample at 400 nm shows a strong quenching (Supplementary Material: Fig. S3). The observed luminescence quenching indicates that there is a strong interaction between the excited state of Ce6 and GO in the hybrid. The quenching may be attributed to two possible competitive processes: photo-induced electron transfer (PET) and energy transfer (ET) [41-44].

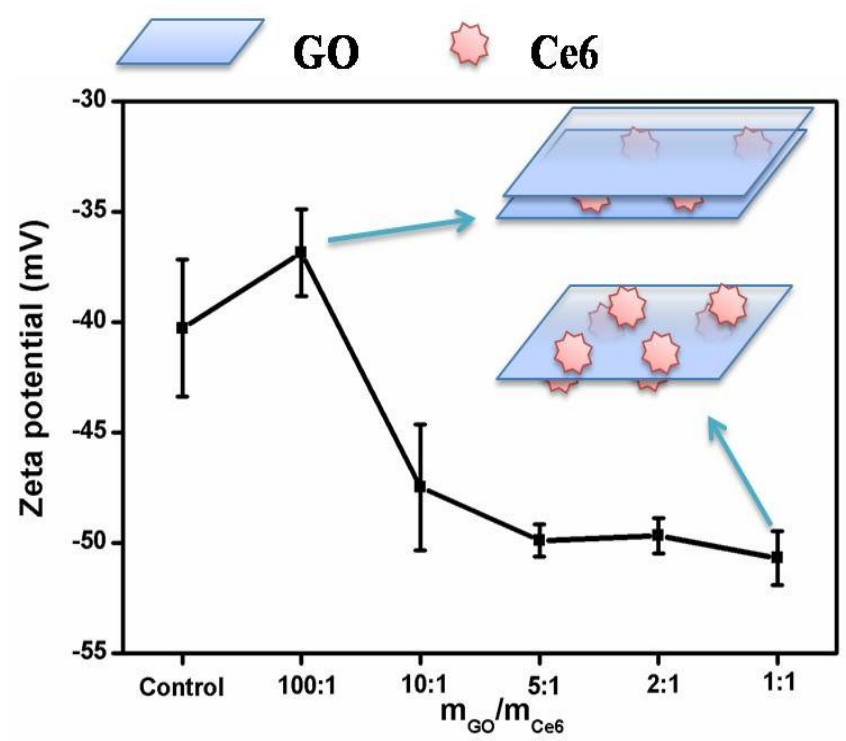

Fig. 4 Changes of the zeta potential of the GO-Ce6 composite suspensions as a function of the component ratio and illustration of formation the GO-Ce6 composite.

\section{Loading Efficiency Measurements}

The loading efficiency of Ce6 was calculated by using Ce6 UV calibration curve at $663 \mathrm{~nm}$. The correlation between the UV absorbance at $663 \mathrm{~nm}$ and the concentration of Ce6 was normalized by linear regression, which showed a well-correlated linear relationship $\left(R^{2}=0.9948\right)$. The standard curve had a good linear relation, described by the following typical equation: $Y=0.07712+27.86047 x$ [14]. The loading efficiency was $\sim 80 \%$, estimated by the typical equation. FA-GO shows a higher PSs loading efficiency, compared to other PSs carriers, such as polymeric micelles and silica nanoparticles [45], which can be contributed to the large specific surface area of GO (two accessible sides). The results suggested that FA-GO as nanocarriers of Ce6 is a very promising approach for PDT.

\section{Intracellular distribution}

We evaluated the cellular selective uptake of FA-GO-Ce6 with a MGC803 cell in RPMI-1640 medium without FA, which were carried out contrasted to other two groups: (a) Cells were treated with GO-Ce6 in RPMI-1640 medium without FA; (b) Cells were treated with FA-GO-Ce6 in RPMI-1640 medium with FA. The fluorescence signal of GO-Ce6 was not observed in Fig.5b, which indicates nanocarriers without FA conjugation didn't have the targeting function. The MGC803 cells incubated with FA-GO-Ce6 and free FA did not exhibit a fluorescence signal (Fig.5d), which highly suggests that free FA can block the binding of FA-GO-Ce6 with folate receptors expressed in the tumor cells. After 30-min incubation with FA-GO-Ce6 in RPMI-1640 medium without FA, MGC803 cells displayed an intense homogeneous cytoplasmic red fluorescence around the nucleus (Fig. $5 \mathrm{f}$ ), indicating selective accumulation of nanocarriers in tumor cells. The strong fluorescence intensity of intracellular FA-GO-Ce6 predicts available PDT effects, because that PDT damage depends on the uptake of Ce6 by tumor cells.

To further investigation on the site of FA-GO-Ce6 in MGC803 cells, CRi Nuance multispectral imaging system was used to detect the fluorescence emission spectra of $\mathrm{Ce} 6$, corresponding to the different sites in the cells. In Fig. 6a, Curve 4 is the standard fluorescence emission spectrum of $\mathrm{Ce} 6$ in the cells with maximum emission peak at $\sim 670 \mathrm{~nm}$. In Fig. $6 b$, site of 1,2 and 3 display the place without cells, the nucleus, and the cytoplasm, respectively. From Fig. 6a, curve 1 indicates that the autofluorescence has a maximum emission peak at $\sim 560 \mathrm{~nm}$. Curve 2 is similar with curve 1 , which suggests that there is no fluorescence signal in the nucleus. Curve 3 has two characteristic peaks, a maximum at $\sim 560 \mathrm{~nm}$, 
which corresponds to autofluorescence, and a shoulder at $\sim 670 \mathrm{~nm}$, which is attributed to Ce6. These re- sults prove that there are abundant of FA-GO-Ce6 in the cytoplasm.

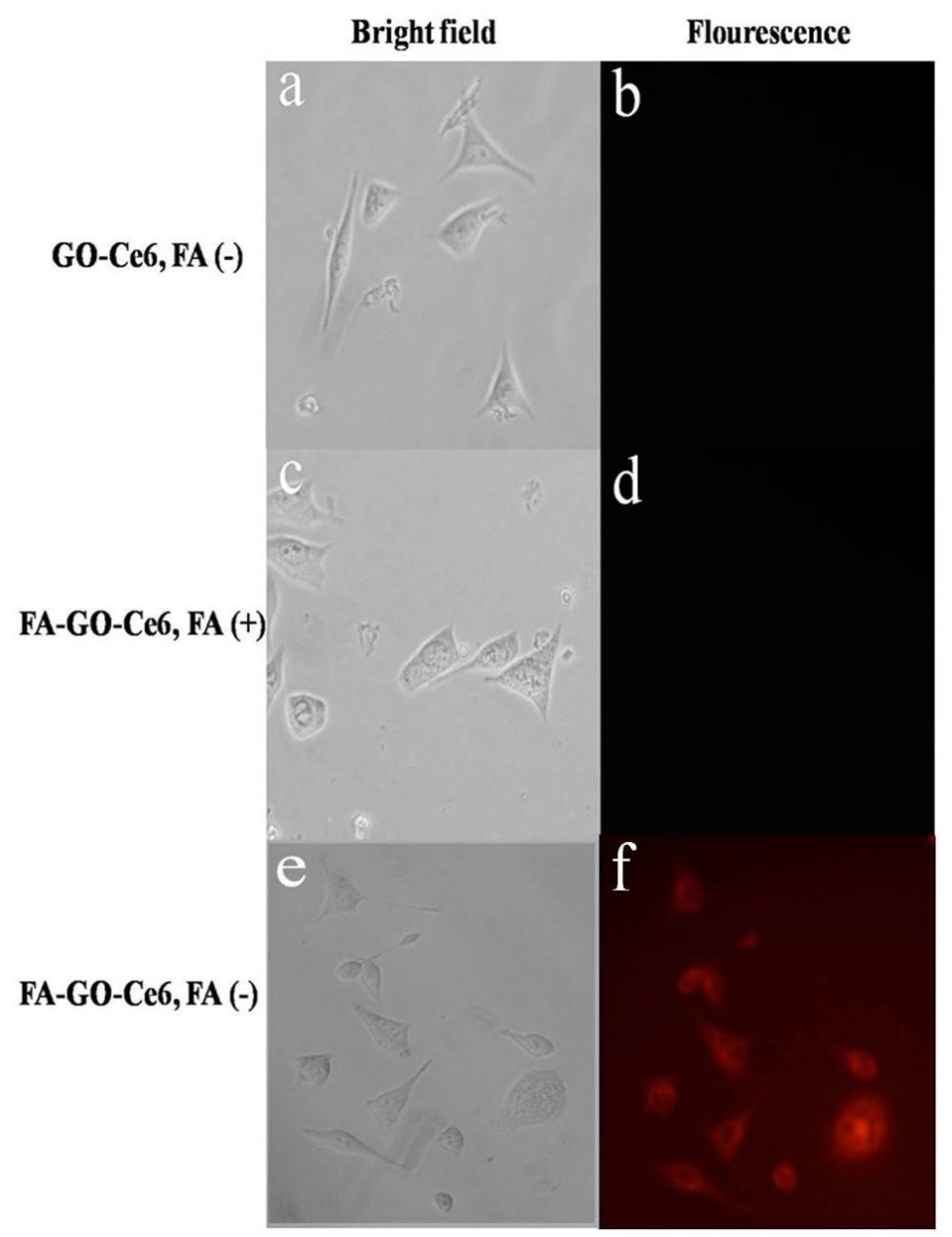

Fig. 5 Selective uptake of GO-Ce6 and FA-GO-Ce6 in MGC803 cells at 30 min incubation of $50 \mu g / m L$ different nanocarriers (left: bright field, right: fluorescence). (a,b) Cells were treated with GO-Ce6 in RPMI-1640 medium without FA. (c,d) Cells were treated with FA-GO-Ce6 in RPMI-I 640 medium with FA. (e,f) Cells were treated with FA-GO-Ce6 in RPMI-I640 medium without FA. All cells monitored by fluorescence microscopy.
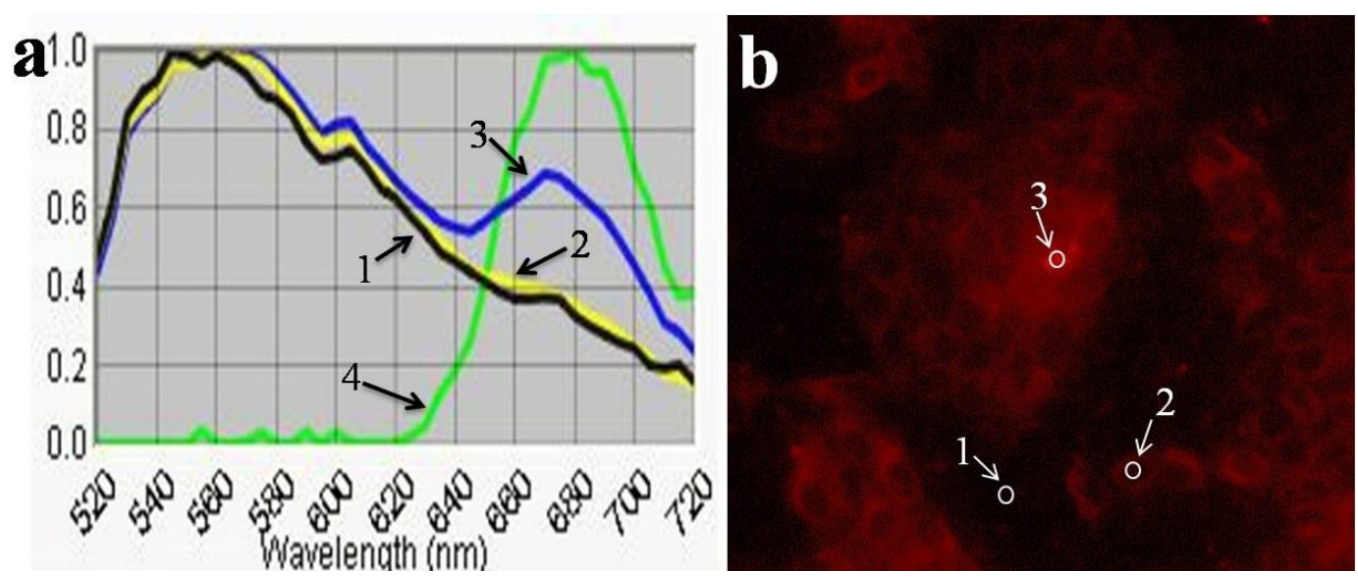

Fig. 6 The typical fluorescence emission spectra (a) and fluorescence image (b) of Cells treated with FA-GO-Ce6 in RPMI-1640 medium without FA. 
For comparison, the ultrastructure of MGC803 cells was also observed by TEM. As shown in Fig. 7, we found that the FA-GO-Ce6 was located both on the surface and in the cytoplasm of MGC803 cells. Meanwhile, there is no FA-GO-Ce6 in the nucleus. The results are consistent with the fluorescence images and spectra (Fig. 5 and 6). To the control group, no nanocarries can be observed in the cells (Supple- mentary Material: Fig. S4). The FA-GO-Ce6 was internalized by cells (Fig. $7 \mathrm{a}$ and $\mathrm{b}$ ) and transferred by vesicles (Fig. $7 \mathrm{c}$ and $\mathrm{d}$ ). Subsequently, some FA-GO-Ce6 entered lysosomes. We infer that FA-GO-Ce6 targeted the FA-receptors, and internalized by receptor-mediated endocytosis through the deformation of the membrance, and then dispersed in the cytoplasm of MGC803 cells [46-47].



Fig. 7 TEM images of MGC803 cells incubated with $100 \mu \mathrm{L}$ of FA-GO-Ce6 $(50 \mu \mathrm{g} / \mathrm{mL})$ for $24 \mathrm{~h}$. (a,c) Low magnification; (b,d) High magnification. Arrows denote the FA-GO-Ce6. "pm" and "num" stand for plasma membrane and nuclear membrane.

\section{Cytotoxicity assessment}

The cytotoxicity of FA-GO and FA-GO-Ce6 was evaluated via classic MTT assays. After 24-h incubation with MGC803 cells, we found that FA-GO possessed non-toxicity. Regarding the effects of FA-GO-Ce6 on MGC803 cells, the ratio of $\mathrm{m}_{\mathrm{FA}-\mathrm{GO}} / \mathrm{m}_{\mathrm{Ce} 6}$ above 2:1 exhibited no dark toxicity, cell viability are more than $80 \%$. When the ratio of $\mathrm{m}_{\mathrm{FA}-\mathrm{GO}} / \mathrm{m}_{\mathrm{Ce} 6}$ reach to $1: 1$, cell viability is less than $50 \%$, the sample exhibited obvious cytotoxicity. These results indicated that the toxicity depends on the concentration of Ce6.

\section{In vitro PDT effect}

The combination of $24 \mathrm{~h}$ exposure of tumor MGC803 cells to FA-GO-Ce6 and laser irradiation induced a drug concentration-dependent cytotoxicity to MGC803 cells, which was statistically significantly different from nonirradiated controls as shown in Fig. 8 . The nonirradiated group displayed more over $80 \%$ 
cell viability, indicating that the FA-GO-Ce6 had no effects on tumor cells without light exposure. To the irradiated group, the ratio of $\mathrm{m}_{\mathrm{FA}-\mathrm{GO}} / \mathrm{m}_{\mathrm{Ce} 6}$ at 2:1 caused approximately $90 \%$ loss of cell viability, demonstrating an obvious photodynamic efficacy. These data suggest that the FA-GO-Ce6 nanocarrier has a high possibility for PDT.

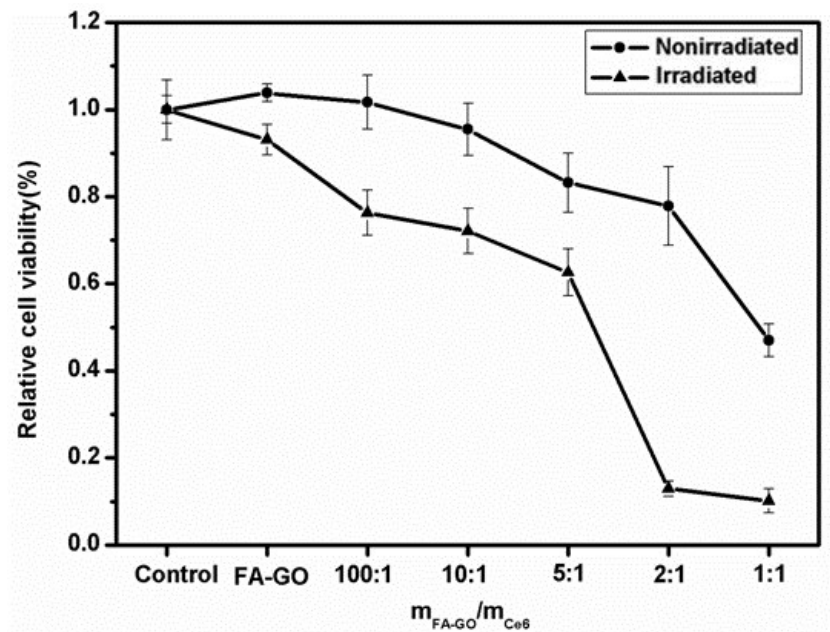

Fig. 8 In vitro photodynamic efficacy of FA-GO-Ce6. MGC803 cells were incubated with 0-100 $\mu$ M FA-GO-Ce6 for $24 \mathrm{~h}$ at $37^{\circ} \mathrm{C}$ in the dark prior to irradiation for $10 \mathrm{~min}$ with 632.8-nm He-Ne laser. Cell viability was determined by MTT assay. Data represent mean \pm SD $(n=3) . P<0.05$ for nonirradiated group versus irradiated group.

\section{Discussion}

According to the results mentioned above, we suggest one possible interaction mechanism between FA-GO-Ce6 and MGC803 cells, shown in Scheme 3 (Fig. C). The whole process includes three steps: (1) The FA-GO-ce6 was endocytosed into cytoplasm and formed endosomes via the folate receptors mediated pathway; (2) When the endosomes were gradually turned into lysosomes, Ce6 was released from FA-GO-Ce6 due to the change of microenvironment in lysosomes ( $\mathrm{pH} 4 \sim 5$ ); (3) the released Ce6 got away from the lysosomes into the cytosol. Subsequently, the photodynamic efficacy was achieved upon irradiation of appropriate wavelength and dosage.

We have demonstrated that a novel drug delivery system based on FA-conjugated GO could be developed with good solubility and low cytotoxicity for targeting PDT. The PS Ce6 has been effectively loaded into the system via hydrophobic interactions and п-п stacking. The nanocarriers can significantly increase the accumulation of PS in tumor cells and lead to a remarkable photodynamic effect on MGC803 cells.
The results indicate the potential applications of FA-conjugated GO in targeting PDT.

Further development of GO-based drug delivery system, the size of GO should be controlled, and the surface of GO should be modify to prevent fluorescence quenching of PSs with GO and improve the photodynamic effect. Currently, we have successfully prepared poly ( $\mathrm{N}$-vinyl pyrrolidone) modified nanoscale GO $(<50 \mathrm{~nm})$ as PS carriers, which are ongoing to assess for in vivo imaging and targeting PDT in our lab. Additionally, due to the strong optical absorbance of GO in the near-infrared region, GO have been used for photo-thermal therapy (PTT) with low-power NIR laser [31]. Based on our studies, if the GO-based PDT combined with PTT, could bring novel opportunities to the next generation of combined cancer treatment. With the irradiation of the single wavelength laser, dual-model therapy platform is desirable development and has great potential in the biomedical application.

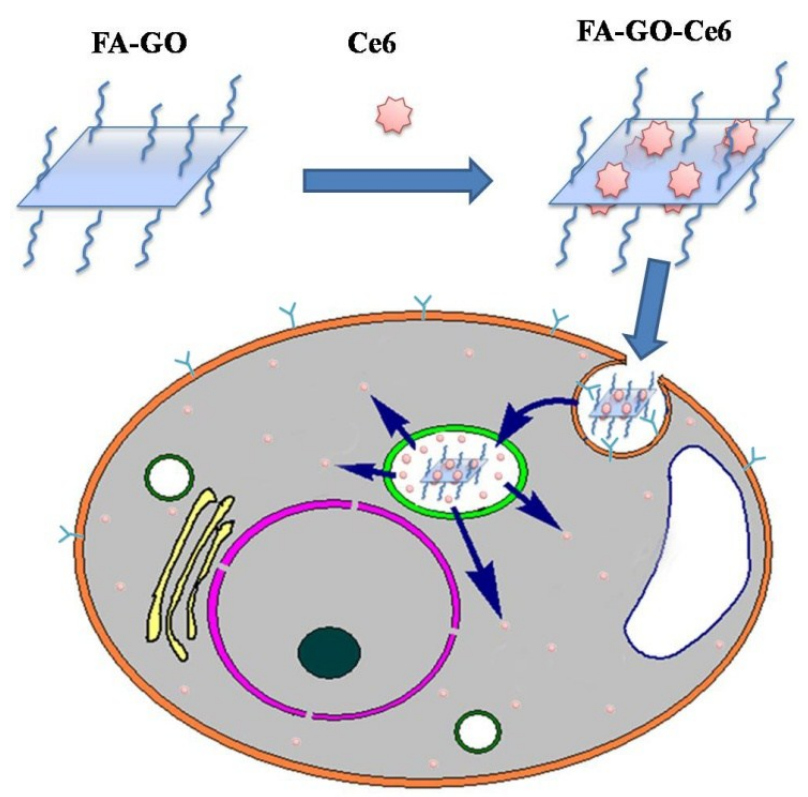

Fig. C. Scheme 3. FA-GO-Ce6 endocytosed by MGC803 cells, endosome-containing FA-GO-Ce6 complexes were located around the nucleus, $\mathrm{Ce} 6$ escaped from the endosome into the cytoplasm.

\section{Acknowledgement}

This work is supported by the National Key Basic Research Program (973 Project) (2010CB933901), National 863 Hi-tech Project (2007AA022004), Important National Science \& Technology Specific Projects (2009ZX10004-311), National Natural Scientific 
Fund (No.20803040), Special project for nano-technology from Shanghai (No.1052nm04100), New Century Excellent Talent of Ministry of Education of China (NCET-08-0350), Shanghai Science and Technology Fund (10XD1406100) and Shanghai Jiao Tong University Innovation Fund for Postgraduates.

\section{Supplementary Material}

Fig. SI FT-IR spectrum of GO.

Fig. S2 Raman spectrum of GO.

Fig. S3 Fluorescence emission spectra of GO-Ce6 composite suspensions (The excitation wavelength is $400 \mathrm{~nm}$ ).

Fig. S4 TEM image of MGC803 cells without nanocarriers.

http://www.thno.org/v0 Ip0240s I.pdf

\section{Conflict of Interest}

The authors have declared that no conflict of interest exists.

\section{References}

1. Huang Z, Xu H, Meyers A, Musani A, Wang L, Tagg R, et al. Photodynamic therapy for treatment of solid tumors"Cpotential and technical challenges. Technol Cancer Res T. 2008;7:309-20.

2. Lovell J, Liu T, Chen J, Zheng G. Activatable Photosensitizers for Imaging and Therapy. Chem Rev. 2010;110:2839-57.

3. Celli J, Spring B, Rizvi I, Evans C, Samkoe K, Verma S, et al. Imaging and Photodynamic Therapy: Mechanisms, Monitoring, and Optimization. Chem Rev. 2010;110:2795-838.

4. Dougherty T, Gomer C, Jori G, Kessel D, Korbelik M, Moan J, et al. Photodynamic therapy. J Natl Cancer I. 1998;90:889-905.

5. Macdonald I, Dougherty T. Basic principles of photodynamic therapy. J Porphyr Phthalocya. 2001;5:105-29.

6. Bonnett R. Photosensitizers of the porphyrin and phthalocyanine series for photodynamic therapy. Chem Soc Rev. 1995;24:19-33.

7. McCaughan J. Photodynamic therapy: a review. Drugs \& Aging. 1999;15:49-68.

8. Guillemard V, Saragovi H. Taxane-antibody conjugates afford potent cytotoxicity, enhanced solubility, and tumor target selectivity. Cancer Res. 2001;61:694.

9. Liu C. Research and development of nanopharmaceuticals in China. Nano Biomed Eng. 2009;1:1-12.

10. Wu R. Advance and Prospect of Nanoscale Chinese Medicine. Nano Biomed Eng. 2010;2:193-200.

11. Bechet D, Couleaud P, Frochot C, Viriot M, Guillemin F, Barberi-Heyob M. Nanoparticles as vehicles for delivery of photodynamic therapy agents. Trends Biotechnol. 2008;26:612-21.

12. Wang S, Gao R, Zhou F, Selke M. Nanomaterials and singlet oxygen photosensitizers: potential applications in photodynamic therapy. J Mater Chem. 2004;14:487-93.

13. Sun Y, Chen Z, Yang X, Huang P, Zhou X, Du X. Magnetic chitosan nanoparticles as a drug delivery system for targeting photodynamic therapy. Nanotechnology. 2009;20:135102.

14. Huang $\mathrm{P}, \mathrm{Li} \mathrm{Z}$, Lin J, Yang $\mathrm{D}$, Gao G, Xu C, et al. Photosensitizer-conjugated magnetic nanoparticles for in vivo simultaneous magnetofluorescent imaging and targeting therapy. Biomaterials. 2011;32:3447-58.
15. Panchapakesan B. Nanotechnology: Part 2 Tiny Technology-Tremendous Therapeutic Potential. Oncology Issues. 2005;20:20-3.

16. Levi-Polyachenko N, Carroll D, Stewart J. Applications of Carbon-Based Nanomaterials for Drug Delivery in Oncology. Medicinal Chemistry and Pharmacological Potential of Fullerenes and Carbon Nanotubes. 2008;:223-66.

17. Huang $\mathrm{P}$, Zhang $\mathrm{C}, \mathrm{Xu} \mathrm{C}$, Bao L, Li Z. Preparation and Characterization of Near-infrared region absorption enhancer carbon nanotubes hybridmaterials. Nano Biomed Eng. 2010;2:231-8.

18. Ajima K, Yudasaka M, Murakami T, Maigne A, Shiba K, Iijima S. Carbon nanohorns as anticancer drug carriers. Mol Pharm. 2005;2:475-80.

19. Mroz P, Tegos G, Gali H, Wharton T, Sarna T, Hamblin M. Fullerenes as photosensitizers in photodynamic therapy. Medicinal Chemistry and Pharmacological Potential of Fullerenes and Carbon Nanotubes. 2008;:79-106.

20. Mroz P, Tegos G, Gali H, Wharton T, Sarna T, Hamblin M. Photodynamic therapy with fullerenes. Photochem Photobiol Sci. 2007;6:1139-49.

21. Zhu Z, Tang Z, Phillips J, Yang R, Wang H, Tan W. Regulation of singlet oxygen generation using single-walled carbon nanotubes. J Am Chem Soc. 2008;130:10856-7.

22. Huang $\mathrm{P}$, Lin J, Yang D, Zhang C, Li Z, Cui D. Photosensitizer-loaded dendrimer-modified multi-walled carbon nanotubes for photodynamic therapy. J Control Release, 2010 , in press.

23. Zhang M, Murakami T, Ajima K, Tsuchida K, Sandanayaka A, Ito $\mathrm{O}$, et al. Fabrication of $\mathrm{ZnPc} /$ protein nanohorns for double photodynamic and hyperthermic cancer phototherapy. Proc Natl Acad Sci U S A. 2008;105:14773-8.

24. Wang K, Ruan J, Song H, Zhang J, Wo Y, Guo S, et al. Biocompatibility of Graphene Oxide. Nanoscale Res Lett. 2010;:1-8.

25. Chen D, Tang L, Li J. Graphene-based materials in electrochemistry. Chem Soc Rev. 2010;39:3157-80.

26. Sun X, Liu Z, Welsher K, Robinson J, Goodwin A, Zaric S, et al. Nano-graphene oxide for cellular imaging and drug delivery. Nano Res. 2008:1:203-12.

27. Liu Z, Robinson J, Sun X, Dai H. PEGylated nanographene oxide for delivery of water-insoluble cancer drugs. J Am Chem Soc. 2008;130:10876-7.

28. Zhang L, Xia J, Zhao Q, Liu L, Zhang Z. Functional Graphene Oxide as a Nanocarrier for Controlled Loading and Targeted Delivery of Mixed Anticancer Drugs. Small. 2010;6:537-44.

29. Zhang F, Zheng B, Zhang J, Huang X, Liu H, Guo S. Horseradish Peroxidase Immobilized on Graphene Oxide: Physical Properties and Applications in Phenolic Compound Removal. J Phys Chem C. 2010;114:8469-73.

30. Yang X, Zhang X, Liu Z, Ma Y, Huang Y, Chen Y. High-efficiency loading and controlled release of doxorubicin hydrochloride on graphene oxide. J Phys Chem C. 2008;112:17554-8

31. Yang K, Zhang S, Zhang G, Sun X, Lee S, Liu Z. Graphene in Mice: Ultrahigh In Vivo Tumor Uptake and Efficient Photothermal Therapy. Nano Lett. 2010;10:3318-23.

32. Zhang J, Yang H, Shen G, Cheng P, Guo S. Reduction of graphene oxide vial-ascorbic acid. Chem Commun (Cambridge, England). 2010;46:1112-4.

33. Hummers Jr W, Offeman R. Preparation of graphitic oxide. J Am Chem Soc. 1958;80:1339.

34. Park S, Ruoff R. Chemical methods for the production of graphenes. Nat Nanotechnol. 2009;4:217-24.

35. Johnsson B, Lofas S, Lindquist G. Immobilization of proteins to a carboxymethyldextran-modified gold surface for biospecific 
interaction analysis in surface plasmon resonance sensors. Anal Biochem. 1991;198:268-77.

36. He C, Zhang L, Wang H, Zhang F, Mo X. Physical-chemical Properties and in vitro Biocompatibility Assessment of Spider Silk, Collagen and Polyurethane Nanofiber Scaffolds for Vascular Tissue Engineering. Nano Biomed Eng. 2009;1:80-8.

37. Cui D, Han Y, Li Z, Song H, Wang K, He R, et al. Fluorescent magnetic nanoprobes for in vivo targeted imaging and hyperthermia therapy of prostate cancer. Nano Biomed Eng. 2009;1:61-74.

38. Chen F, Huang P, Mo XM. Electrospinning of Heparin Encapsulated P (LLA-CL) Core/Shell Nanofibers. Nano Biomed Eng. 2010;2:84-90.

39. Choi Y, Weissleder R, Tung C. Selective antitumor effect of novel protease-mediated photodynamic agent. Cancer Res. 2006;66:7225-9.

40. Park K, Lee S, Kang E, Kim K, Choi K, Kwon I. New generation of multifunctional nanoparticles for cancer imaging and therapy. Adv Funct Mater. 2009;19:1553-66.

41. Xu Y, Liu Z, Zhang X, Wang Y, Tian J, Huang Y, et al. A graphene hybrid material covalently functionalized with porphyrin: synthesis and optical limiting property. Adv Mater. 2009;21:1275-9.

42. Karousis N, Economopoulos S, Sarantopoulou E, Tagmatarchis N. Porphyrin counter anion in imidazolium-modified graphene-oxide. Carbon. 2010;48:854-60.

43. Zhang X, Feng Y, Tang S, Feng W. Preparation of a graphene oxide-phthalocyanine hybrid through strong [pi]-[pi] interactions. Carbon. 2010;48:211-6.

44. Geng J, Kong B, Yang S, Jung H. Preparation of graphene relying on porphyrin exfoliation of graphite. Chem Commun (Cambridge, England). 2010;46:5091-3.

45. Dong HQ, Zhao ZL, Wen HY, Li YY, Guo FF, Shen AJ, et al. Poly (ethylene glycol) conjugated nano-graphene oxide for photodynamic therapy. Sci China Ser B Chem. 2010;53:2265-71.

46. Zhou Y, Wang X. Study on synergistic effect of new functionalized $\mathrm{Ag}$ nanoparticles for intracellular drug uptake in cancer cells. Nano Biomed Eng. 2010;2:211-7.

47. Tian F, Prina-Mello A, Estrada G, Beyerle A, M ller W, Schulz $\mathrm{H}$, et al. Macrophage cellular adaptation, localization and imaging of different size polystyrene particles. Nano Biomed Eng. 2009;1:13-26. 